\title{
Tuning of flow control loop using DeltaV DCS and MATLAB
}

\author{
Rohit Nagvekar \\ Department of Instrumentation \& \\ Control Engineering Vishwakarma Institute of \\ Technology, \\ Pune. \\ Shubhangi Gajdhane \\ Department of Instrumentation \& Control Engineering \\ Vishwakarma Institute of Technology, \\ Pune
}

\author{
Abhiraj Bhalerao \\ Department of Instrumentation \& Control Engineering \\ Vishwakarma Institute of Technology, \\ Pune.
}

Prof. Vijaykumar Bhanuse

Department of Instrumentation \& Control Engineering Vishwakarma Institute of Technology,

Pune

\section{Prasad Doifode \\ Department of Instrumentation \& Control Engineering \\ Vishwakarma Institute of Technology,}

Pune.

\begin{abstract}
Process management instrumentality is meant to work the plants on a gentle state basis and square measure most significant to work out the performance of automatic system. management of the method needs thorough management data, the behavior of the method parts, method details, and inter-relation at intervals the sections of the method plant. Flow management encompasses a big selection of application in method industries. In $90 \%$ of method management applications have a tendency to manipulate the flow to get desired output. The aim work centered on pelvic inflammatory disease standardization methodology and flow management loop implementation. Flow is dynamic parameter thus totally different standardization strategies for standardization pelvic inflammatory disease viz and ZieglerNichols standardization methodology square measure thought-about. dominant flow victimization DCS is that the best combination. DCS provides advanced management ways to regulate the method parameters. thus we have a tendency to square measure attending to interface flow management loop with DCS. Study its behavior for a step input modification verify its transfer operate of our model victimization advanced DCS management functions. The comparative studies show that the simulations results vary from real time implementation results.
\end{abstract}

Keywords- "PID tuning, Ziegler Nichols, Internal Model control, MATLAB, DeltaV DCS".

\section{INTRODUCTION}

Distributed control system, is a computerized control system for a process or plant usually with many control loop, in which autonomous controllers are distributed throughout the system, but there is no central operator supervisory control. This is in contrast to systems that use centralized controllers. Discrete controllers located at central control room or within a central computer. The DCS concept increases the reliability and reduces the installation cost by localizing the control functions near the process plant, with remote monitoring and supervision. Today the DCS and SCADA systems are very similar, but DCS tends to be used on a large scale process plant where high reliability and security is important. The processors receive information from input modules, process the information and decide control actions to be signaled by the output modules. The field inputs and outputs can be analog signals e.g. 4-20 mA DC current loop or two-state signals that switch either ON or OFF, such as relay contacts or a semiconductor switch. DCS has separate cards for DI, DO, $\mathrm{AI}, \mathrm{AO}$ and each card having 8channels.

\section{LITERATURE SURVEY}

Maduri Jarang implemented PID Tuning of Level Control loop using DeltaV DCS and concluded PID tuning using IMC gives best response for FOPDT process model.

Sujit Anandrao Jagnade in his paper "Modeling, Simulation and Control of Flow Tank System" described various approaches on how to determine Process Transfer Function involving Flow Control loop using ZN method, Relay Auto tuner etc. [2]

J.S Rajashekar in his paper "Flow Control Loop Analysis for System Modeling\& Identification" studied \&analyzed flow control loop components, where by experimentation carried out on an industrial type control valve, I/P converter, orifice in the laboratory. Effective data collection of these components, consisting of literature gathering and some tests such as step and vibration on these components, is performed using which modeling equations are formed in MATLAB. [3]

H. Šiljak in his paper "An Extended Model of a Level and Flow Control System" providing an improved transfer function model of this system's level and flow control loops Results are obtained using MATLAB System Identification Toolbox after data acquisition in Lab VIEW [4].

\section{PROCESS SETUP}

The process hardware setup is shown in the given Fig. 1 The flow control loop components are as: air filter 
regulator, pneumatic control valve, vortex flow meter. Flow transmitter. The pressure required for control valve is 3 to $15 \mathrm{psig}$. In this system capacitance type flow transmitter is used to measure the flow of tank $24 \mathrm{~V}$ power supply is required for flow transmitter it is a four wire transmitter. And output of transmitter is $4-20 \mathrm{~mA}$.

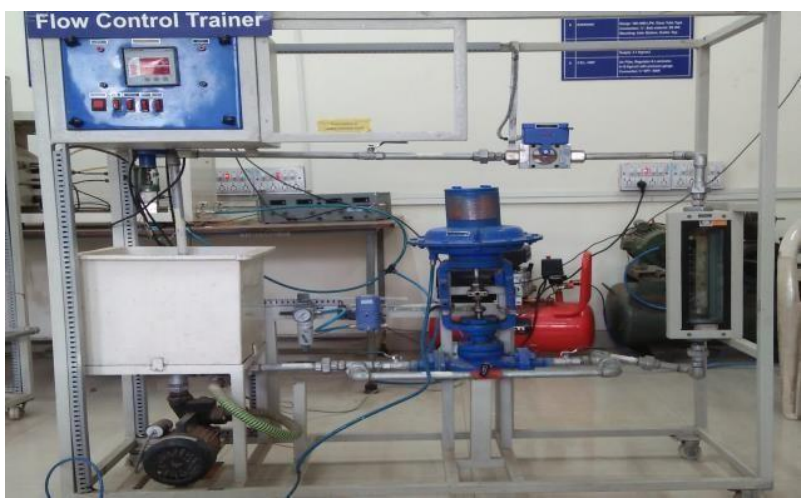

Fig 1. Process setup.

\section{SYSTEM DESCRIPTION}

As shown in fig. 2 below, flow control loop system is basically a feedback control system. The flow from the pneumatic control valve is given to the turbine flow transmitter which converts the input flow into 4-20ma signal. This 4-20mA signal is then given to the DCS system with the help of twisted pair cable that carries $4-20 \mathrm{~mA}$ signal. The DCS involves a PID controller functional block which takes care of making the controlled variable closer to the set point value. It does so by calculating the values for proportional gain, reset and rate parameters by setting the mode of the controller in the auto state. The values for proportional gain, rate and reset are calculated using Ziegler Nichols tuning method. In this way DCS will make sure that the controlled variable which in this case is flow reaches closer to the set point value. Other tuning methods like Internal model control were also considered. The output of DCS goes as an input to the current to pressure converter which held responsible to close or open the control valve depending upon the output of the DeltaV DCS. In this way, the flow inside the loop will said to be controlled as per the requirement or set point value.

The block diagram of the proposed system is as follows.

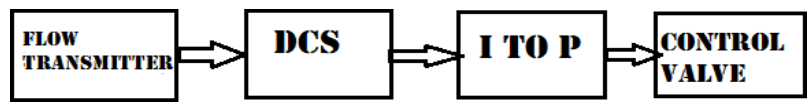

Fig 2. Block diagram of the system

\section{SYSTEM IDENTIFICATION}

This is one of the powerful but complex controller mode combining proportional, integral and derivative control modes. It has following advantages:

(a) One-to-one correspondence between $\mathrm{P}$ and ep.

(b) Eliminates offset of P-mode.

(c) Provides fast response.
Mathematically, PID controller is written as:

$\mathrm{P}=\mathrm{K}_{\mathrm{p}} \cdot \mathrm{e}_{\mathrm{p}}+\mathrm{K}_{\mathrm{p}} \cdot \mathrm{KI}_{\mathrm{I}} \int^{\mathrm{t}} \mathrm{e} \mathrm{pdt}+\mathrm{K}_{\mathrm{p}} \mathrm{KD}(\mathrm{dep} / \mathrm{dt})+\mathrm{PI}(0)$

where,

$\mathrm{K} \mathrm{p}=$ Proportional gain

$\mathrm{KI}$ = Integral gain

$\mathrm{Kd}=$ Derivative gain

$\mathrm{Kp}$ is expressed in terms of proportional band $\mathrm{PB}$, $\mathrm{KI}$ is expressed in terms of integral time $\mathrm{TI}$, and $\mathrm{KD}$ is expressed in terms of derivative time TD. For better control PB should be less, TI should be less and TD should be high. PID controller can be used as $\mathrm{P}$ controller by removing I and $\mathrm{D}$ actions. Integral action can be removed by using $\mathrm{T} \mathrm{I}=$ maximum. Derivative action can be removed by using $\mathrm{TD}=0$. Similarly, PID controller can be used as PI and PD controller, by removing $\mathrm{D}$-action $(\mathrm{Td}=0)$ and I-action $(\mathrm{TI}$ = maximum) respectively.

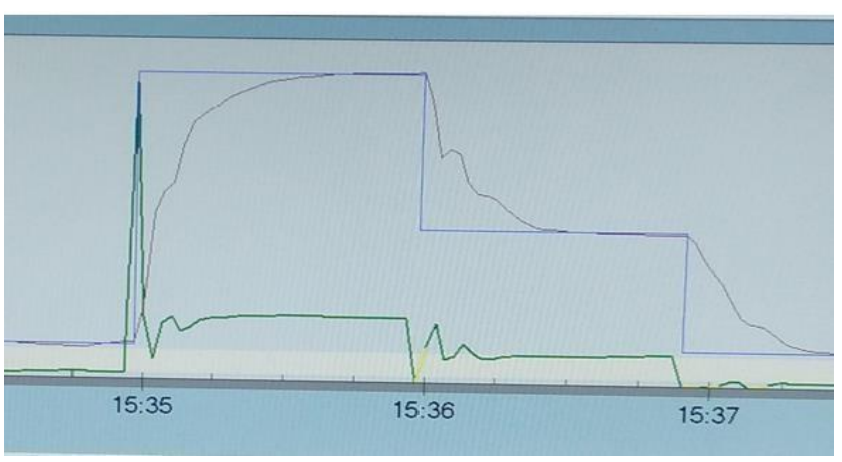

Fig 3. Step response of the system

This graph is recorded in process history view in DeltaV of real time experimentation in open loop. To find the transfer function of open loop system above graph is used. Calculate the transfer function by $63.3 \%$ approach of process variable. Following is the transfer function of the system:

$$
T F=\frac{0.45}{9.5 s+1}
$$

After finding out the transfer function, the values of Kp, Tp are obtained and finding out the PID tuning parameters in MATLAB, using following method.

\section{Internal Model Control (IMC):}

The IMC method, like the DS method is based on an assumed process model and leads to analytical expressions for the controller setting. These two design methods are closely related and produce identical controllers if the design parameters are specified in a consistent manner. 


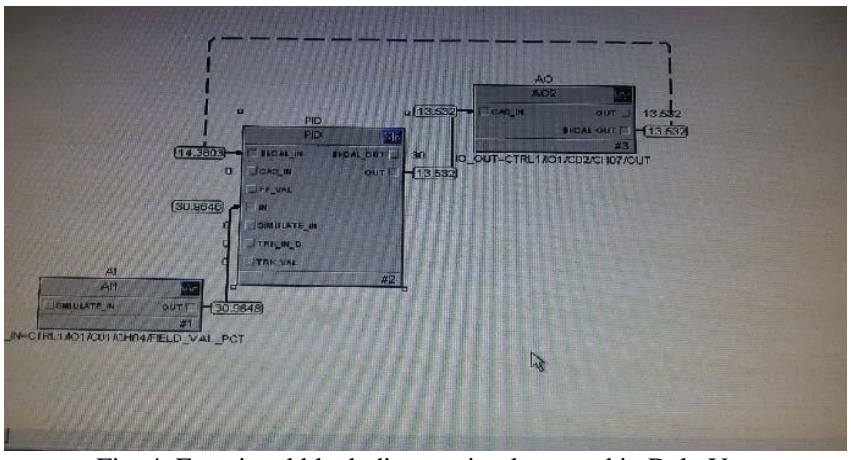

Fig. 4. Functional block diagram implemented in DeltaV

\section{Analog Input block}

Analog Input Block uses only single analog measurement value and status from an I/O channel. Transmitter's 4$20 \mathrm{~mA}$ signal cab be taken as input value. Generally, we use $\mathrm{CH}-2$ of analog input card for the flow transmitter.

\section{Analog Output block}

Analog Output Block is used to give an analog output value to a field device with the help of a specified I/O channel or Field bus device. Generally, $\mathrm{CH}-3$ of analog output card is used to provide the output of control valve.

\section{PID block}

PID block gathers all the necessary logic required to perform proportional-integral- derivative (PID) control, analog input channel processing and analog output channel processing in a single function. The following figure shows the PID tuning parameters of IMC in PID parameters block

\section{RESULTS AND ANALYSIS}

1.Step response for IMC using the values calculated theoretically showed better result as shown in the table and helps to meet the required specifications. The output response for IMC in auto tuner in MATLAB is shown in the fig.5.1 below

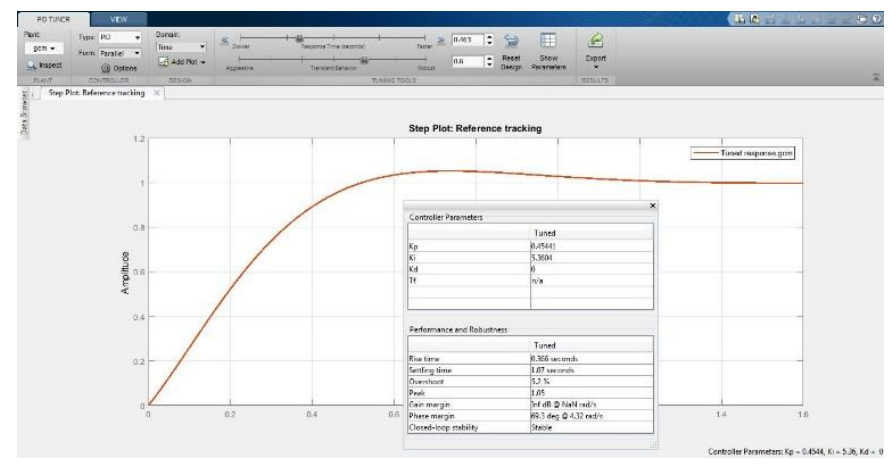

Fig 5.1 Output for IMC

2.Step response for PID auto tuner (MATLAB tool) showed

slow response comparatively with much higher rise time and settling time. The values are mentioned in the table. The step response using PID auto tuner is as shown in the figure 5.2 below

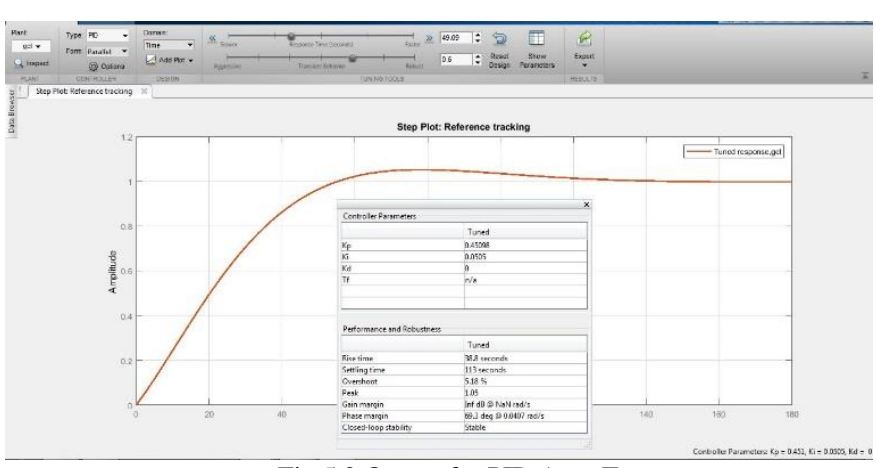

Fig 5.2 Output for PID Auto Tuner

3. PID tuning using trial and error method for flow control loop using DeltaV insight showed the following result in fig. 5.3, using this, the values settling time and rise time are obtained and is shown in table,

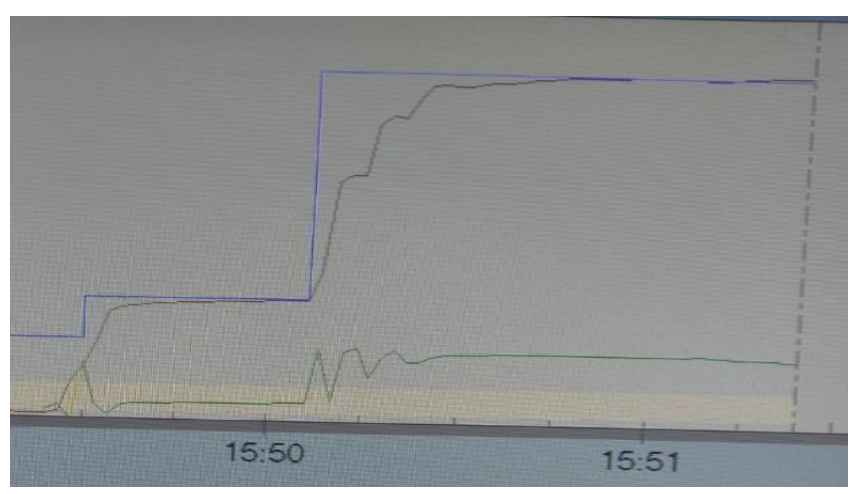

Fig 5.3 PID Tuning using DeltaV Insight

The following table shows the values obtained from the responses,

\begin{tabular}{|c||c|c|c|}
\hline Parameter & PID & IMC & $\begin{array}{c}\text { DeltaV } \\
\text { Insight }\end{array}$ \\
\hline \hline $\mathrm{Kp}$ & 0.45 & 0.45 & 0.45 \\
\hline $\mathrm{Ki}$ & 0.05 & 5.3604 & 4.9 \\
\hline $\mathrm{Kd}$ & 0 & 0 & 0 \\
\hline $\mathrm{Ts}$ & $113 \mathrm{Sec}$ & $1.07 \mathrm{Sec}$ & $37 \mathrm{Sec}$ \\
\hline $\mathrm{Tr}$ & $38.8 \mathrm{Sec}$ & $0.366 \mathrm{Sec}$ & $0.2 \mathrm{Sec}$ \\
\hline
\end{tabular}

Table No. 1

Keeping the $\mathrm{Kp}$ value constant and $\mathrm{Kd}$ value zero and using trial and error method for PID and IMC technique.

\section{CONCLUSION}

The three tuning methods: Internal Model Controller, PID tuner (MATLAB tool) and the trial and error method are studied with their implementation for flow control loop. The gain values are determined by the trial and error method. As per the simulation results, it seems that the trial and error method showed good response to a step change but the settling time observed was more. The PID tuning, using auto-tuner in MATLAB shows high rise and settling time compared to the IMC technique. By using trial and error method for PID tuning, the flow control loop performs better. In addition, IMC technique should also be considered to improve the system response. 


\section{REFERENCE}

[1] M. Yousefi, T. Binazadeh, M. H. Shafiei "The design of robust fractional order PI controller for liquid flow control of a spherical tank" $3^{\text {rd }}$ International Conference on Control, Instrumentation, and Automation (ICCIA 2013), December 2830,2013, Tehran,Iran

[2] L.Wang, W.R. Cluett, "Tuning PID controllers for integrating processes," IEE Proc.-Control Theory Appl., Vol. 144, No 5, September 1997.

[3] Ramasamy M. and Sundaramoorthy S., "PID controller tuning for desired closed loop responses for SISO systems using impulse response, Computers and Chemical Engineering," 32, 2008, pp. 1773-1788.

[4] Neha S. Narkhede,"'Labview based system for PID tuning and implementation for flow control loop," Department of Instrumentation Vishwakarma Institute of Technology Pune, Maharashtra, India 411037 